\title{
Melanoma ocular em cães: relato de dois
} casos

\section{Ocular melanoma in dogs: report of two cases}

\section{Resumo}

Os melanomas normalmente são neoplasias cutâneas, mas podem ocorrer sempre que agrupamentos de melanócitos são encontrados. Estes tumores possuem duas categorias: melanoma benigno, referido como melanocitoma, e melanoma maligno. O local mais comum da sua ocorrência no cão é a cavidade oral, mas dentre as neoplasias oculares representam a mais comum neoplasia ocular primária nesta espécie. Quanto a sua localização podem ocorrer na conjuntiva, limbo (tecidos epibulbares), úvea anterior, coróide e nos anexos oculares. A localização anatômica destas neoplasias é de grande importância para a determinação do protocolo terapêutico mais adequado e consequentemente do seu prognóstico.

\section{Summary}

Melanomas are usually skin cancers, but may occur when clusters of melanocytes are found. These tumors have two classes: benign melanoma, referred to as melanocytoma, and malignant melanoma. The most common site of it's occurrence is in the dog's mouth, but among ocular tumors represent the most common primary eye cancer in this species. Their location could be in the conjunctiva, limbus (epibulbar tissues), anterior uvea, choroid and eye annexes. The anatomical location of these neoplasms is of great importance to determine the most appropriate treatment protocol and therefore their prognosis. 
Ana Paula Teixeira da Silva ${ }^{1}$

Marina Lie Sawada²

Alessandra Oliveira Pinheiro²

Maria Lúcia Marcucci Torres ${ }^{3}$

Priscila Carvalho de Oliveira Balieiro ${ }^{4}$
Ana Paula Teixeira da Silva

Rua Daniel de Oliveira Carvalho, 825

13660-000, Porto Ferreira - SP, Brasil

z +551992085760

vet.anapaula@gmail.com

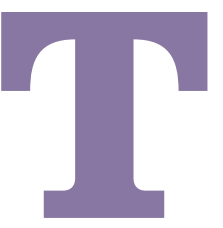

umores de origem melanocítica representam a mais comum neoplasia ocular primária em cães e, nesta espécie, são mais frequentes que em qualquer outra (CANPOLAT; YAMAN; GUNAY, 2007; CAVALCANTE, 2006; DA CONCEIÇÃO et al., 2010; DITERS et al., 1983; DUBIELZIG, 2002; KLEINER; SILVA; MASUDA, 2003; WILCOCK, 2009). Apresentam duas categorias: melanoma benigno, referido como melanocitoma e melanoma maligno (DUBIELZIG, 2002; KLEINER; SILVA; MASUDA, 2003; WILCOCK, 2009).

Os melanomas normalmente são neoplasias cutâneas, mas podem ocorrer sempre que agrupamentos de melanócitos são encontrados (CANPOLAT; YAMAN; GUNAY, 2007). O local mais comum da sua ocorrência no cão é a cavidade oral, com alta probabilidade de metástases (CAVALCANTE, 2006; INTILE, 2010).

Nos melanomas oculares há quatro localizações possíveis: conjuntiva, limbo (tecidos epibulbares), úvea anterior e coróide (BAPTISTA, 2003). A úvea anterior, íris e corpo ciliar são os locais mais acometidos, já a coróide é raramente atingida (BAPTISTA, 2003;

1 Residente da área de Clínica Médica e Reprodução de Pequenos Animais da Faculdade de Medicina Veterinária do Centro Universitário da Fundação de Ensino Octávio Bastos - UNIFEOB, São João da Boa Vista - São Paulo, Brasil.

2 Residente da área de clínica médica e reprodução de pequenos animais da Faculdade de Medicina Veterinária do Centro Universitário da Fundação de Ensino Octávio Bastos - UNIFEOB, São João da Boa Vista - São Paulo, Brasil.

3 Professora Ms. da Faculdade de Medicina Veterinária do Centro Universitário da Fundação de Ensino Octávio Bastos - UNIFEOB, da disciplina de clínica médica de pequenos animais, semiologia veterinária e medicina preventiva - São João da Boa Vista - São Paulo, Brasil.

4 Professora Ms. e Dra. da Faculdade de Medicina Veterinária do Centro Universitário da Fundação de Ensino Octávio Bastos - UNIFEOB, da disciplina de reprodução e obstetrícia animal - São João da Boa Vista - São Paulo, Brasil.disciplina de clínica médica de pequenos animais, semiologia veterinária e medicina preventiva - São João da Boa Vista - São Paulo, Brasil. 
CANPOLAT; YAMAN; GUNAY, 2007; CAVALCANTE, 2006; DA CONCEIÇÃO et al., 2010; DUBIELZIG, 2002; SIMON, 2008; TEIXEIRA; BARROS; BARROS, 2007). Nos anexos oculares há propensão para as conjuntivas da membrana nictitante, palpebral e bulbar (BAPTISTA, 2003; DA CONCEIÇÃO et al., 2010; DELGADO, 2011).

Geralmente os melanomas acometem cães mais velhos, a partir de oito anos, a questão racial não está bem definida, e alguns autores referem uma maior incidência nos machos (CAVALCANTE, 2006; DUBIELZIG, 2002; KLEINER; SILVA; MASUDA, 2003).

Dentre os sinais clínicos o paciente poderá apresentar uveíte não responsiva, hifema, opacidade corneana, endoftalmite por necrose tumoral, massa tumoral visível, glaucoma (KLEINER; SILVA; MASUDA, 2003), em alguns casos a principal queixa é o sangramento agudo ocular (ENGLISH, 2006). Também pode ocorrer hemorragia intra-ocular, descolamento de retina, infiltração para o nervo óptico e conseqüentemente cegueira (KLEINER; SILVA; MASUDA, 2003).

No melanoma maligno, a capacidade de causar sinais sistêmicos é lenta. No entanto, ocorrem danos extensos em estruturas oculares adjacentes, como invasão de esclera e órbita, elevando-se, assim, as chances de ocorrerem metástases (DUBIELZIG, 2002; KLEINER; SILVA; MASUDA, 2003). Os tumores primários podem produzir metástases pela via sanguínea ou linfática (BAPTISTA, 2003; DA CONCEIÇÃO et al., 2010). Neste caso, os órgãos acometidos são a tireóide (SIMON, 2008), pulmões, fígado, cérebro, rins (CANPOLAT; YAMAN; GUNAY, 2007; CARVALHO, 2005; CAVALCANTE, 2006), coração e baço (ZAMBONI et al., 2011). Contudo, nas neoplasias intra-oculares as metástases são raras, exceto nos casos de melanoma oral nos cães (DUBIELZIG, 2002).

As neoplasias oculares secundárias são raras, devido ao pequeno tamanho do tecido ocular (DUBIELZIG, 2002). Os tumores com potencial metástatico mais comum para o globo ocular dos cães são linfoma, hemangiossarcoma, adenocarcinoma mamário e melanoma oral maligno (DA CONCEIÇÃO et al., 2010; DUBIELZIG, 2002; TEIXEIRA; BARROS; BARROS, 2007).

Dentre os métodos diagnósticos para as neoplasias oculares há a oftalmoscopia indireta, ultrassonografia, angiografia fluoresceínica e a gonioscopia (BAPTISTA, 2003; KLEINER; SILVA; MASUDA, 2003; SIMON, 2008). Após a identificação do tumor pode-se lançar mão de outros métodos diagnósticos como a citologia e/ ou histopatológico que permitem assegura se realmente existe um processo neoplásico que possa ser classificado (CAVALCANTE, 2006). Os possíveis diagnósticos diferenciais incluem as proliferações cistos iridociliares
(DELGADO, 2011; KLEINER; SILVA; MASUDA, 2003), alterações granulomatosas, metástases de neoplasias extra-oculares e prolapso de íris (KLEINER; SILVA; MASUDA, 2003).

Para o tratamento de melanomas oculares podem ser utilizadas placas radioativas, termoterapia transpupilar, enucleação, exenteração, quimioterapia ou imunoterapia, dependendo das características do mesmo (ARCIERI et al., 2002).

O tratamento de tumores em anexos oculares consiste na remoção cirúrgica completa, quando diagnosticado precocemente (DA CONCEIÇÃO et al., 2010; DELGADO, 2011). Nos casos em que o tumor não pode ser completamente removido ou já houve metástase, pode-se optar pela radioterapia (INTILE, 2010), com taxa de êxito de $50 \%$, mas as recidivas são altas (CARVALHO 2005; INTILE, 2010). Sobre a quimioterapia, os melanomas apresentam resistência a drogas quimioterápicas, e as taxas de resposta e duração não têm sido satisfatórias, e também não foi observado benefício na sobrevida dos cães (CALVACANTE, 2006; INTILE, 2010; TEIXEIRA; BARROS; BARROS, 2007).

A terapia mais recente é a imunoterapia, uma vacina para melanoma oral canino. A vacina é administrada a cada 15 dias, em um total de quatro doses, depois reforços são administrados a cada seis meses para o resto da vida. A imunoterapia é usada com um adjuvante no tratamento, não sendo usada como terapia única. Seus efeitos colateriais são raros e foi constatada melhora na expectativa de vida dos animais (INTILE, 2010).

A localização anatômica das neoplasias oculares, sobretudo as de origem melanocíticas, é de grande importância para a determinação do protocolo terapêutico mais adequado e do prognóstico (CARVALHO, 2005; DELGADO, 2011).

\section{RELATO DE CASO}

\section{Caso clínico 1}

No Hospital Veterinário da Fundação de Ensino Octávio Bastos, foi atendido um cão sem raça definida, de aproximadamente seis anos, apresentando vermelhidão no olho esquerdo e dificuldade visual.

No exame físico, foram observados: hifema no olho esquerdo e opacidade circunscrita de coloração acinzentada na córnea em ambos os olhos, compatível com Florida spots. Devido ao hospital não dispor de um oftalmologista em período integral no atendimento hospitalar, o paciente foi atendido pelo setor de clínica médica, onde foi realizado hemograma, que apresentou trombocitopenia, neutrofilia, eosinopenia e monocitopenia. 


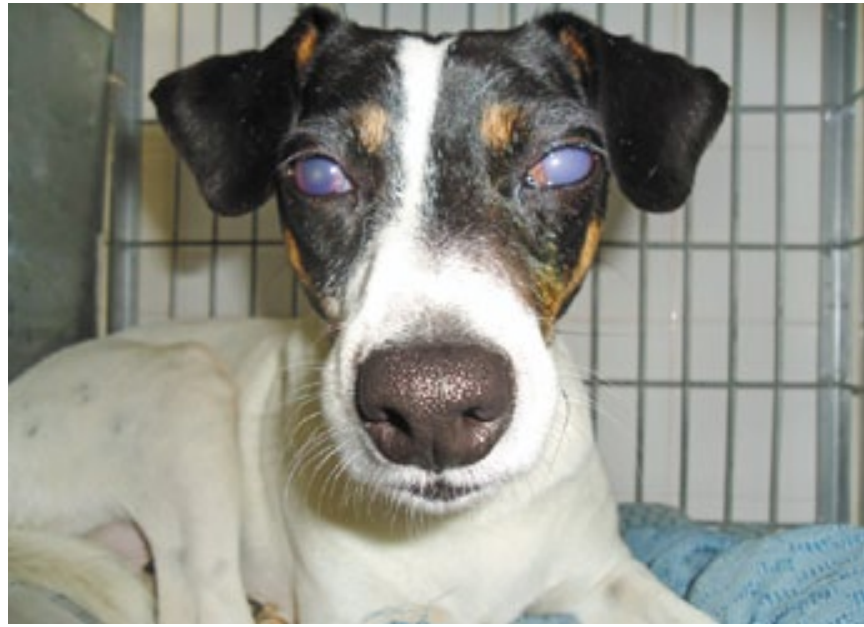

Figura 1 - Evidência do edema de córnea bilateral

Fonte: Arquivo próprio.

Mediante o quadro oftalmológico e a diminuição no número de plaquetas, alterações compatíveis com quadro de hemoparasitose, foi instituído o tratamento com doxiciclina $(10 \mathrm{mg} / \mathrm{kg}$, via oral, a cada 12 horas, por 28 dias), atropina $(0,44 \mathrm{mg} / \mathrm{kg}$, via subcutânea, duas aplicações com intervalo de 15 dias), dipropionato de imidocarb $(5 \mathrm{mg} / \mathrm{kg}$, via subcutânea, duas aplicações com intervalo de 15 dias), e para uveíte, colírio a base de tobramicina e dexametasona (a cada seis horas, via ocular, por 10 dias).

Após 13 dias, o paciente retornou ao Hospital Veterinário, apresentando uma piora do quadro clínico geral, no qual foram constatados: anorexia, dor, e perda total da capacidade visual. No exame físico apresentava: hifema em olho esquerdo, edema corneal bilateral (figura 1), reflexos direto e consensual diminuídos em ambos os olhos e ejeção de vasos na esclera também em ambos os olhos. Á palpação foi observado um possível aumento da pressão intra-ocular, e devido a esta suspeita, foi solicitada a mensuração da pressão intra-ocular e, iniciou-se o tratamento antiglaucomatoso com prednisolona $1 \%$ colírio (a cada seis horas, via ocular, por período indeterminado), timolol $0,5 \%$ colírio e brinzolamida $1 \%$ colírio (ambos a cada oito horas, via ocular, por período indeterminado).

Diante do novo quadro clínico do paciente, com intervalo de 15 dias da primeira consulta, foi realizado o exame ultrassonográfico que detectou massa em globo ocular esquerdo, com possível compressão do nervo óptico e descolamento de retina no olho direito. Aferiu-se a pressão ocular, cujo resultado foi acima dos valores de normalidade que correspondem de $15-30 \mathrm{mmHg}$, os resultados do paciente foram de $68 \mathrm{mmHg}$ no olho esquerdo e $54 \mathrm{mmHg}$ no olho direito, apresentando aumento da pressão intraocular mesmo sob terapia para glaucoma, não havendo assim, resposta ao tratamento instituído.

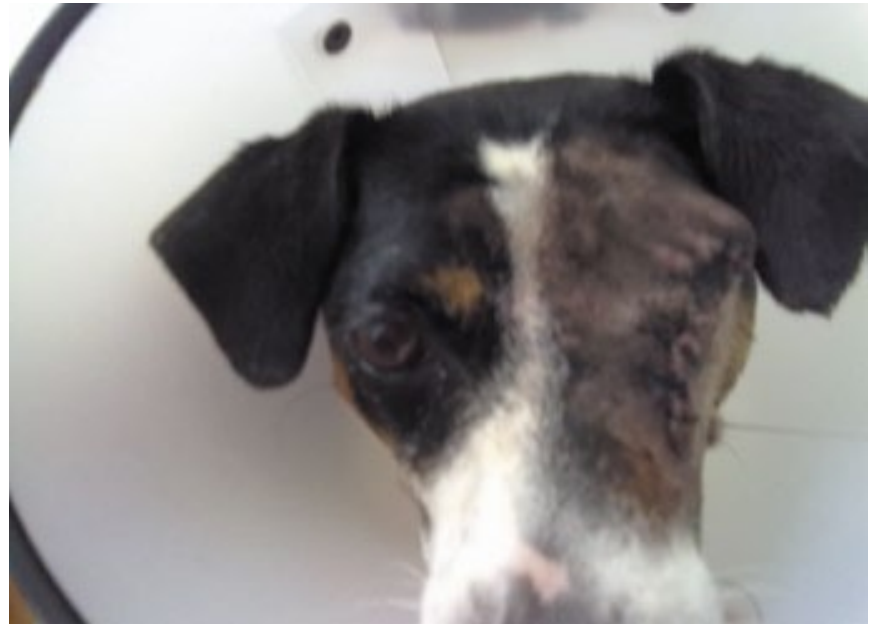

Figura 2 - Avaliação clínica dias depois da intervenção cirúrgica Fonte: Arquivo próprio.

Mediante o quadro clínico do paciente, que apresentava apatia, anorexia, muita dor á palpação em região ocular; e dos resultados obtidos no exame ultrassonográfico e tonomeria, optou-se para realização da exenteração do globo ocular esquerdo e; atrofia iatrogênica do globo ocular direito com administração intra-coular de $1 \mathrm{ml}$ de gentamicina e $1 \mathrm{ml}$ de dexametasona (figura 2). Foi também realizado exame histopatológico do material coletado, cujo resultado foi melanoma em região de estroma do corpo ciliar (figura 3).

A terapia instituída no pós-operatorio foi: cloridrato de tramadol $(5 \mathrm{mg} / \mathrm{kg}$, via oral, a cada 8 horas, por 5 dias), cefalexina $(30 \mathrm{mg} / \mathrm{kg}$, via oral, a cada 12 horas, por 15 dias), meloxicam $(0,1 \mathrm{mg} / \mathrm{kg}$, via oral, a cada 24 horas, por 5 dias) e limpeza local com solução fisiológica $0,9 \%$.

Após os procedimentos cirúrgicos, o animal apresentava-se bem clinicamente. Por se tratar de um tumor maligno, foi indicado quimioterapia com doxorrubicina $\left(30 \mathrm{mg} / \mathrm{m}^{2}\right.$, via intravenosa, seis aplicações com intervalo de 21 dias), mas a proprietária negou-se a realizar o tratamento. (referência já descrita na discussão)

Em aproximadamente um mês de pós-operatório, o olho direito não havia atrofiado e apresentava intenso edema corneal, dor e bulftalmia. Portanto, foi realizado um novo ultrasson ocular que revelou massa intra-vítrea no olho direito. $\mathrm{O}$ exame radiográfico destinado a procura de metástase, foi negativo. No entanto, houve um aumento da silhueta cardíaca, compatível com cardiopatia, sendo assim instituído o tratamento com maleato de enalapril $(0,5 \mathrm{mg} /$ $\mathrm{kg}$, via oral, a cada 12 horas, por período indeterminado).

Diante da confirmação de massa em olho direito o paciente foi encaminhado para realização da exenteração do globo ocular direito e histopatologia da massa, que confirmou melanoma intra-ocular. 

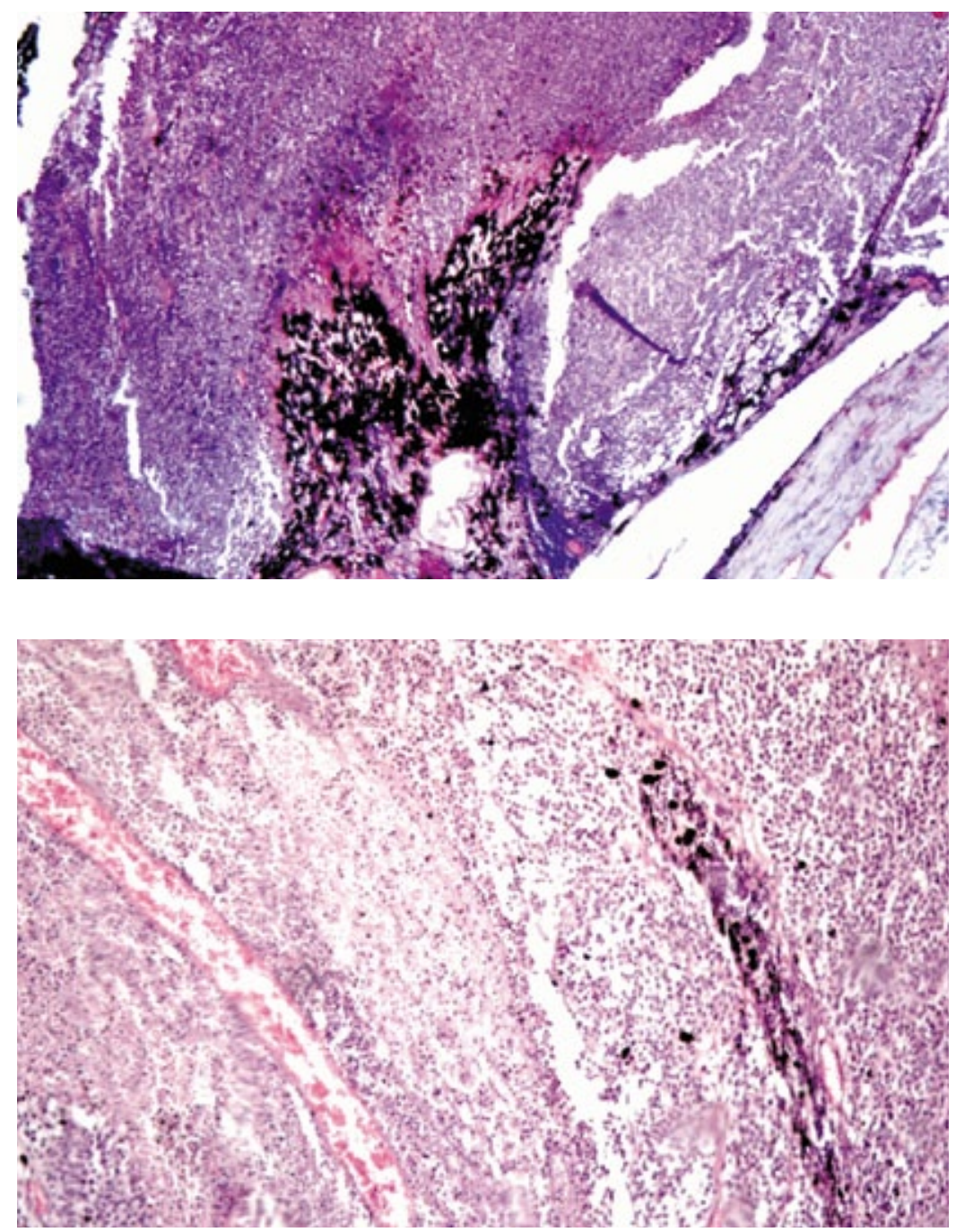

Figura 3 - Fotomicroscopia do globo ocular esquerdo com melanoma, $5 x$ Fonte: arquivo próprio.
Figura 4 - Fotomicroscopia do globo ocular esquerdo com melanoma, $20 \mathrm{x}$

Fonte: Arquivo próprio.
O protocolo pós-cirúrgico instituído foi: cefalexina $(30 \mathrm{mg} / \mathrm{kg}$, via oral, a cada 12 horas, por 15 dias), meloxicam $(0,1 \mathrm{mg} / \mathrm{kg}$, via oral, a cada 24 horas, por 5 dias $)$, cloridrato de tramadol $(5 \mathrm{mg} / \mathrm{kg}$, via oral, a cada 8 horas, por 5 dias), ranitidina $(2 \mathrm{mg} / \mathrm{kg}$, via oral, a cada 12 horas, por 15 dias) e limpeza local com solução fisiológica $0,9 \%$.

A realização de quimioterapia foi indicada bem como a de um ultrasson abdominal para pesquisa de metástases, mas a proprietária negou-se a realizar o tratamento quimioterápico, porém, aceitou a realização do ultrasson, que não apresentou alterações.

Um mês após o último procedimento cirúrgico, foi realizada a última consulta clínica do paciente, que se apresentava em bom estado clínico geral e adaptado a perda da capacidade visual.

\section{Caso clínico 2}

Foi atendida, no Hospital Veterinário da Fundação de Ensino Octávio Bastos, uma cadela da raça Cocker Spaniel de 12 anos de idade, em que o proprietário relatava que há 20 dias havia observado uma pele sobre o olho direito do animal, com rápida evolução. A paciente já se encontrava sob tratamento com colírio a base de dexametasona, neomicina, polimixina B (a cada 24 horas, via ocular).

Ao exame físico foram observadas as alterações: edema na pálpebra inferior direita, conjuntiva palpebral inferior direita edemaciada, hiperêmica e prolapsada, epífora bilateral, opacificação bilateral do cristalino (figura 5). Na oftalmoscopia indireta, o olho direito, apresentou ausência de coloração tapetal, papila edemaciada, com contorno irregular e de coloração acinzentada. Também foram constatados valores abaixo do normal no Teste de schirmer, sendo os resultados 3 $\mathrm{mm}$ no olho direito e $12 \mathrm{~mm}$ no olho esquerdo, cujo valores de referência são de $15-25 \mathrm{~mm}$. 


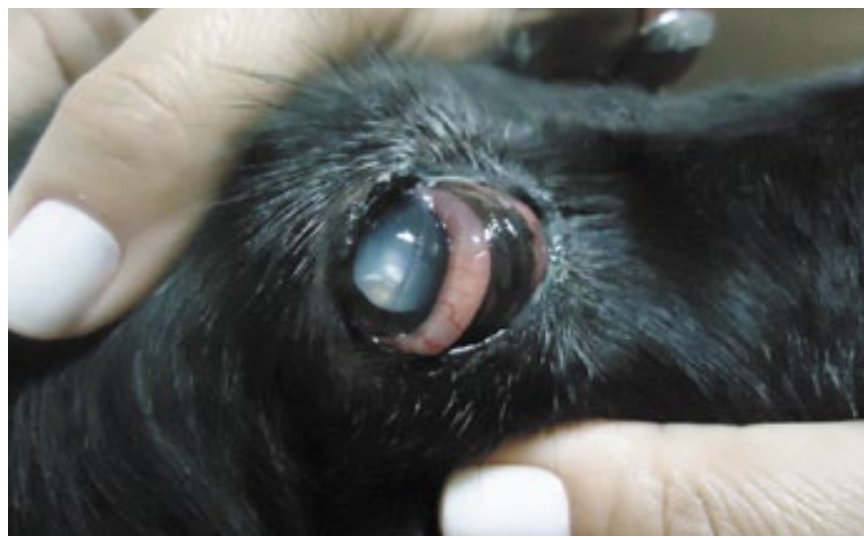

Figura 5 - Globo ocular direito apresentando conjuntiva palpebral inferior edemaciada hiperêmica e prolapsada, opacificação bilateral do cristalino

Fonte: Arquivo próprio.

Diante destas alterações suspeitou-se de corioretinite e quemose no olho direito, assim foi mantido o colírio a base de dexametasona, neomicina, polimixina B (a cada seis horas, via ocular), e prescrito e tacrolimus $0,03 \%$ (a cada oito horas, via ocular), até a realização de um ultrasson ocular, para auxiliar no diagnóstico.

O exame ultrassonográfico do olho direito revelou catarata e nódulo em margem medial, caracterizando um processo inflamatório. Baseado no laudo ultrassonográfico, houve mudanças no protocolo terapêutico, sendo prescrito prednisolona $(1,5 \mathrm{mg} / \mathrm{kg}$, a cada 12 horas, via oral, por sete dias), tobramicina colírio (a cada seis horas, via ocular, por período indeterminado) e prednisolona $1 \%$ colírio (a cada seis horas, via ocular, por período indeterminado), o tacrolimus $0,03 \%$ (a cada oito horas, via ocular, por período indeterminado) foi mantido.

Em oito dias o paciente retornou ao Hospital Veterinário e apresentava um aumento da massa em região ocular (figura 6), sendo realizado exame citológico do nódulo localizado na conjuntiva palpebral direita, onde o resultado foi de melanoma maligno.

Baseado neste resultado, foi indicado o tratamento cirúrgico: exenteração do globo ocular direito e a realização de exame radiográfico a procura de metástase, que revelou massa no pulmão. Mediante este fato, não foi realizado o procedimento cirúrgico indicado; mantendose o tratamento já instituído, destinado a oferecer uma melhor qualidade de vida ao paciente, que apresentava um bom quadro clinico geral.

Em apenas quatro dias, o paciente retornou ao Hospital Veterinário apresentando sinais neurológicos (convulsões focais), aumento significativo da massa e intensa secreção ocular purulenta bilateral. Devido à rápida evolução do quadro o desconforto em que o mesmo se encontrava, foi realizada a sua eutanásia.

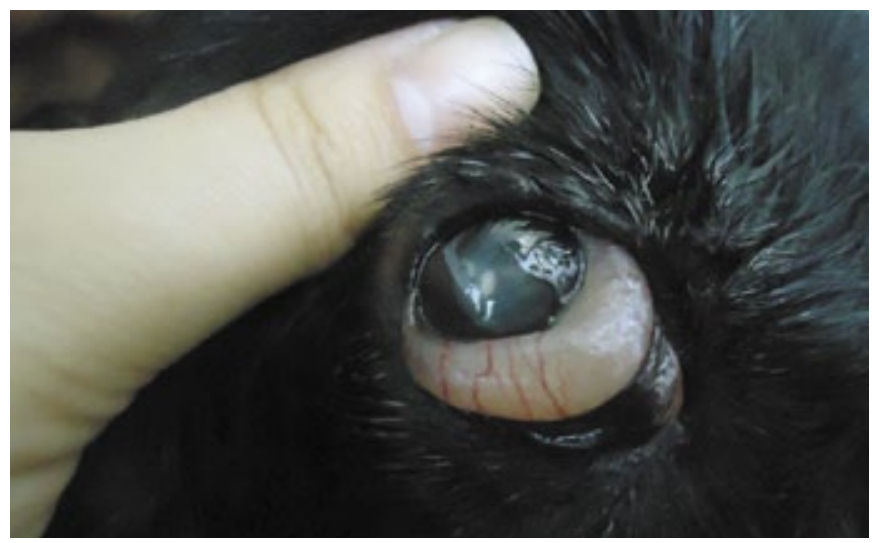

Figura 6 - Globo ocular direito após oito dias de tratamento, apresentando aumento do edema da conjuntiva palpebral

Fonte: Arquivo próprio.

\section{Discussão}

Como já descrito, os melanomas normalmente são neoplasias cutâneas, mas podem ocorrer sempre que agrupamentos de melanócitos são encontrados (CANPOLAT; YAMAN; GUNAY, 2007). Neste trabalho foram descritos dois casos de melanoma ocular, localizados em regiões citadas na literatura com maior probabilidade de ocorrência; como região da úvea (BAPTISTA, 2003; CANPOLAT; YAMAN; GUNAY, 2007; CAVALCANTE, 2006; DA CONCEIÇÃO et al., 2010; DUBIELZIG, 2002; SIMON, 2008; TEIXEIRA; BARROS; BARROS, 2007) e a conjuntiva palpebral (BAPTISTA, 2003; DA CONCEIÇÃO et al., 2010; DELGADO, 2011).

A prevalência desta neoplasia se dá, em cães a partir de oito anos de idade (DUBIELZIG, 2002; KLEINER; SILVA; MASUDA, 2003). Os animais citados neste trabalho apresentaram idade próxima a relatada, sendo um destes da raça Cocker Spaniel, que é descrita como uma das raças predispostas (CAVALCANTE, 2006).

De acordo com a sua localização, os tumores melanocíticos apresentam características e prognósticos diferentes. No primeiro relato de caso, o cão sem raça definida apresentou melanoma no olho esquerdo na região de estroma do corpo ciliar; geralmente, estes tumores apresentam massa elevada, pigmentação da íris, crescimento lento, localmente invasivo e baixo potencial metastático (BAPTISTA, 2003). Kleiner, Silva e Masuda (2003) referiram que os melanomas benignos e malignos, que afetam, principalmente, o corpo ciliar e a coróide, possuem um grande potencial de malignidade, por serem altamente invasivos.

A princípio, o paciente foi submetido ao tratamento para hemoparasitose (Nelson e Couto, 2006), uma vez que os sinais clínicos apresentados foram compatíveis com esta patologia. $\mathrm{O}$ animal também apresentava um 
aumento bilateral da pressão intra-ocular, sendo os valores de referência de $15-30 \mathrm{mmHg}$, portanto foi instituído o tratamento antiglaucomatoso já descrito (MARTINS; RIBEIRO; LAUS, 2007). No entanto, a terapia antiglaucomatosa não foi bem-sucedida e optou-se pela atrofia iatrogênica do globo ocular direito, cuja técnica consiste na drenagem do humor vítreo na região da esclera, seguida da administração na mesma região de $1 \mathrm{ml}$ de gentamicina e $1 \mathrm{ml}$ de dexametasona (PETRILLO; VASCONCELOS; VASCONCELOS, 2006).

Decorrido aproximadamente um mês da realização do procedimento cirúrgico no olho esquerdo, o paciente apresentou aumento de volume no olho direto, sendo constatado no histopatológico melanoma intra-ocular, mas não foi possível a confirmação da região exata da neoplasia, pois, geralmente, os tumores uveais originamse da íris e corpo ciliar, mas quando crescem fica difícil a determinação de sua origem (Barros e Barros, 2007).

Contudo prevalece a dúvida, se o segundo processo neoplásico seria um foco de metástase ou se era um tumor primário. Pois, nas neoplasias oculares em geral, as metástases são raras. No entanto, neste caso, trata-se de um tumor melanocítico dotado de um grande potencial de malignidade por ser altamente invasivo e que por ser capaz de causar um dano extenso nas estruturas oculares adjacentes, como invasão de esclera e órbita, apresenta uma maior chance da ocorrência de metástases (DUBIELZIG, 2002; KLEINER; SILVA; MASUDA, 2003).

No segundo caso, o paciente da raça Cocker Spaniel apresentou melanoma maligno na conjuntiva palpebral. O tumor apresentou características de grande malignidade. Devido à rápida evolução tumoral, sinais clínicos e o diagnóstico de massa no pulmão. Este comportamento tumoral assemelha-se às características clínicas e comportamento biológico de tumores melanocíticos localizados na conjuntiva palpebral. Os tumores localizados nesta região apresentam-se como uma massa elevada, com pigmentação da conjuntiva, geralmente localizamse na membrana nictitante, são de crescimento rápido, localmente agressivos e com elevado potencial recidivo. São potencialmente metastáticos, altamente malignos e possuem comportamento idêntico ao melanoma oral (BAPTISTA, 2003; ENGLISH, 2006).

Em ambos os casos, o tratamento recomendado foi a remoção cirúrgica. No primeiro caso, foi realizada a exenteração dos globos oculares e recomendada a quimioterapia. Conforme Delgado (2011) e Teixeira, Barros e Barros (2007), o tratamento de melanomas na úvea consiste na enucleação ou exenteração do globo ocular, com ou sem exérese de linfonodos associados e eventual quimioterapia. O protocolo quimioterápico recomendado foi a doxorrubicina, $30 \mathrm{mg} / \mathrm{m}^{2}$, com infusão endovenosa durante 10 a 20 minutos, a cada três a quatro semanas, no total de seis aplicações (RODASKI; NARDI, 2004), mas a proprietária negou-se a realizar a quimioterapia.

No segundo caso, não foi possível a realização do procedimento cirúrgico, devido ao animal apresentar metástase pulmonar. Teixeira, Barros e Barros (2007) descrevem que em casos em que, os tumores atingem outras estruturas e o paciente apresenta metástase, síndrome paraneoplásica, caquexia e comprometimento da qualidade de vida, a eutanásia deve ser instituída. Como a paciente também apresentava uma perda na qualidade de vida, a eutanásia foi realizada.

Como citado anteriormente, a localização das neoplasias oculares é de grande importância para se determinar o protocolo terapêutico e o prognóstico (CARVALHO, 2005; DELGADO, 2011). Pacientes tratados cirurgicamente com neoplasias pequenas, apresentam uma sobrevida, em média, de 12 meses e uma porcentagem de óbito de $54 \%$ dentro de dois anos. Já os que apresentam neoplasias grandes possuem uma sobrevida de quatro meses e a porcentagem de óbito é de $100 \%$ dentro de dois anos. No entanto, há autores que relatam que em melanomas intra-oculares a taxa de mortalidade é baixa. (CAVALCANTE, 2006).

\section{Conclusão}

Este trabalho foi escrito no intuito de alertar os médicos veterinários sobre a importância do diagnóstico das neoplasias oculares em cães, sendo as neoplasias melanocíticas as mais comuns nesta espécie. A localização e a precocidade do diagnóstico são de suma importância para a determinação do prognóstico e, assim, oferecer uma melhor qualidade de vida ao paciente. Os melanomas oculares podem assumir características de malignidade idênticas aos de cavidade oral. 


\section{Referências}

1. ARCIERI, E. S.; FONSECA, D.; FRANÇA, E. T.; BRAGA, E. F.; FERREIRA, M. A. Estudo de melanoma de coróide na Universidade Federal de Uberlândia. Arquivo Brasileiro Oftalmologia, v. 65, n. 1, 89-93, 2002.

2. BAPTISTA, C. S. N. F. Cão e gato caracterização ecográfica em modo brilho e modo amplitude de melanomas epibulbares e da uvea anterior. 2003. 107 f. Dissertação (Mestrado) - Instituto de Ciências Biomédicas de Abel Salazar, Universidade do Porto, Porto, 2003. Disponível em: <http://repositorio-aberto.up.pt/ bitstream/10216/9567/3/5270_TM_01_P.pdf>. Acesso em: 26 out. 2011.

3. CANPOLAT, I.; YAMAN, I.; GUNAY, C. A case of primary intraocular malignant iris melanoma in an Akkaraman sheep. Revue Médecine Vétérinaire, v. 158, n. 4, p. 171 $173,2007$.

4. CARVALHO, S. L. M. Melanoma. 2005. 81 f. TCC (Especialização em Clínica Médica e Cirúrgica em Pequenos Animais) - Faculdades Integradas. UPIS, Brasília, 2005. Disponível em: <http://www.upis.br/pesquisas/pdf/veterinaria/Silvia\%20 Luanna\%20Mendes\%20Carvalho.pdf>. Acesso em: 26 out. 2011.

5. CAVALCANTE, J. A. Avaliação da incidência do melanoma em um estudo retrospectivo de 37 cães (Canis familiaris) com neoplasias, atendidos na clínica Escola de Medicina Veterinária da Universidade Castelo Branco e na clínica veterinária Ossian. 2006. 69 f. TCC (Especialização em Clínica Médica e Cirúrgica em Pequenos Animais) Escola de Medicina Veterinária, Universidade Castelo Branco, Rio de Janeiro, 2006. Disponível em: <http://www.qualittas.com.br/documentos/Avaliacao\%20da\%20 Incidencia\%20do\%20Melanoma\%20-\%20Jose\%20Alberto\%20Cavalcante.PDF>. Acesso em: 26 out. 2011

6. DA CONCEIÇÃO, L. F.; RIBEIRO, A. P.; PISO, D. Y. T.; LAUS, J. L. Consideration about ocular neoplasia of dogs and cats. Revista Ciência Rural, v. 40, n. 10 Santa Maria, 2010. Disponível em: <http://www.scielo.br/scielo.php?pid=S0103 84782010001000030\&script=sci_arttext>. Acesso em: 19 jan. 2012.

7. DELGADO, E. Abordagem ás neoplasias oculares - A enucleação é a solucção? In ENCONTRO DE FORMAÇÃO OMV, 2., 2011, Lisboa. 2011.

8. DITERS, R. W.; DUBELZIG, R. R.; AGUIRRE, G. D.; ACLAND, G. M. Primary ocular melanoma in dogs. Veterinary Pathology, v. 20, p. 379-395, 1983.

9. DUBIELZIG, R. R. Tumors of the eye. In: MEUTEN, D. J. Tumors of domestic animals 4. ed. lowa: lowa State, 2002. cap. 15, p. 739-754.

10. ENGLISH, R. Animal eye care associates ocular tumors. [S.I.: s.n.], 2006. p. 1-8 Disponível em: <http://www.dcavm.org/Ocular\%20tumor\%20notes\%202006. pdf>. Acesso em: 26 out. 2011

11. GALERA, D. P.; LAUS, J. L.; ORIÁ, A. P. Afecções da Túnica Fibrosa. In: LAUS, J. L. Oftalmologia clínica e cirúrgica em cães e em gatos. São Paulo: Roca, 2007. cap. 4, p. 69-96.
12. HERREA, D. Afecções da córnea. In: HERRERA, D. Oftalmologia clínica em animais de companhia. São Paulo: MedVet, 2008. cap. 7, p. 111-140.

13. INTILE, J. Canine melanoma and the melanoma vaccine. Quarterly Bulletin Summer, micro News: Veterinary Specialists and Emergency Services (Oncology) 2010. Disponivel em: <http://www.pdfking.com/melanoma-vaccine-and-caninemelanoma-article/>. Acesso em: 26 out. 2011.

14. KLEINER, J. A.; SILVA, E. G.; MASUDA, E. K. Melanoma intra-ocular primário de coróide em um cão da raça Rottweiller: relato de caso. In: CONFERÊNCIA SULAMERICANA DE MEDICINA VETERINÁRIA, 3., 2003, Rio de Janeiro. Anais... Rio de Janeiro, 2003.

15. MARTINS, B. C.; RIBEIRO, A. P.; LAUS, J. L. Glaucoma. In: LAUS, J. L. (Ed.). Oftalmologia clínica e cirúrgica em cães e em gatos. São Paulo: Roca, 2007. cap. 7 , p. 151-167.

16. NELSON, R. W.; COUTO, C. G. Medicina interna de pequenos animais. 3. ed. São Paulo: Elsevier, 2006. p. 1227-1234

17. ORIÁ, A. P.; LAUS, J. L. Tópicos em oftalmologia dos felinos. In: LAUS, J. L. Oftalmologia clínica e cirúrgica em cães e em gatos. São Paulo: Roca, 2007. cap. 9 p. 191-224.

18. PETRILLO, V. H. M.; VASCONCELOS, M. I. C.; VASCONCELOS, M. G. C. Atrofia de globo ocular para tratamento de glaucoma. Revista Científica Eletrônica de Medicina Veterinária, ano 3, n. 6. jan. 2006. Disponível em: <http://www.revista.inf.br/ veterinaria06/relatos/relato01.pdf >. Acesso em: 08 abr. 2013.

19. RODASKI, S.; DE NARDI, A. B. Quimioterapia antineoplásica em cães e gatos. Curitiba: Maio, 2004. cap. 2, p. 32-160.

20.SIMON, M. Afecções do trato uveal. In: HERRERA, D. Oftalmologia clínica em animais de companhia. São Paulo: MedVet, 2008. cap. 10, p. 173-193.

21. TEIXEIRA, A. L.; BARROS, L. F. M.; BARROS, P. S. M. Afecções da túnica vascular. In: LAUS, J. L. Oftalmologia clínica e cirúrgica em cães e em gatos. São Paulo: Roca, 2007. cap. 5, p. 97-110

22. WILCOCK, B. P. Olhos, pálpebras, conjuntiva e órbita. In: MC GAVIN, M. D ZACHARY, J. F. Bases da patologia em veterinária. 4. ed. Rio de Janeiro: Elsevier 2009. Cap. 20, p. 1349-1413.

23.ZAMBONI, R.; WEIBERG, A.; NUNES, C. S.; GEVEHR-FERNANDES, C. Estudo retrospectivo de melanoma canino durante o período de 2002 a 2011. In: CONGRESSO DE INICIAÇÃO CIENTíFICA, 3; MOSTRA CIENTíFICA, 3., 2011, Pelotas. Anais... Pelotas: UFPEL, 2011. 